Document downloaded from:

http://hdl.handle.net/10251/101127

This paper must be cited as:

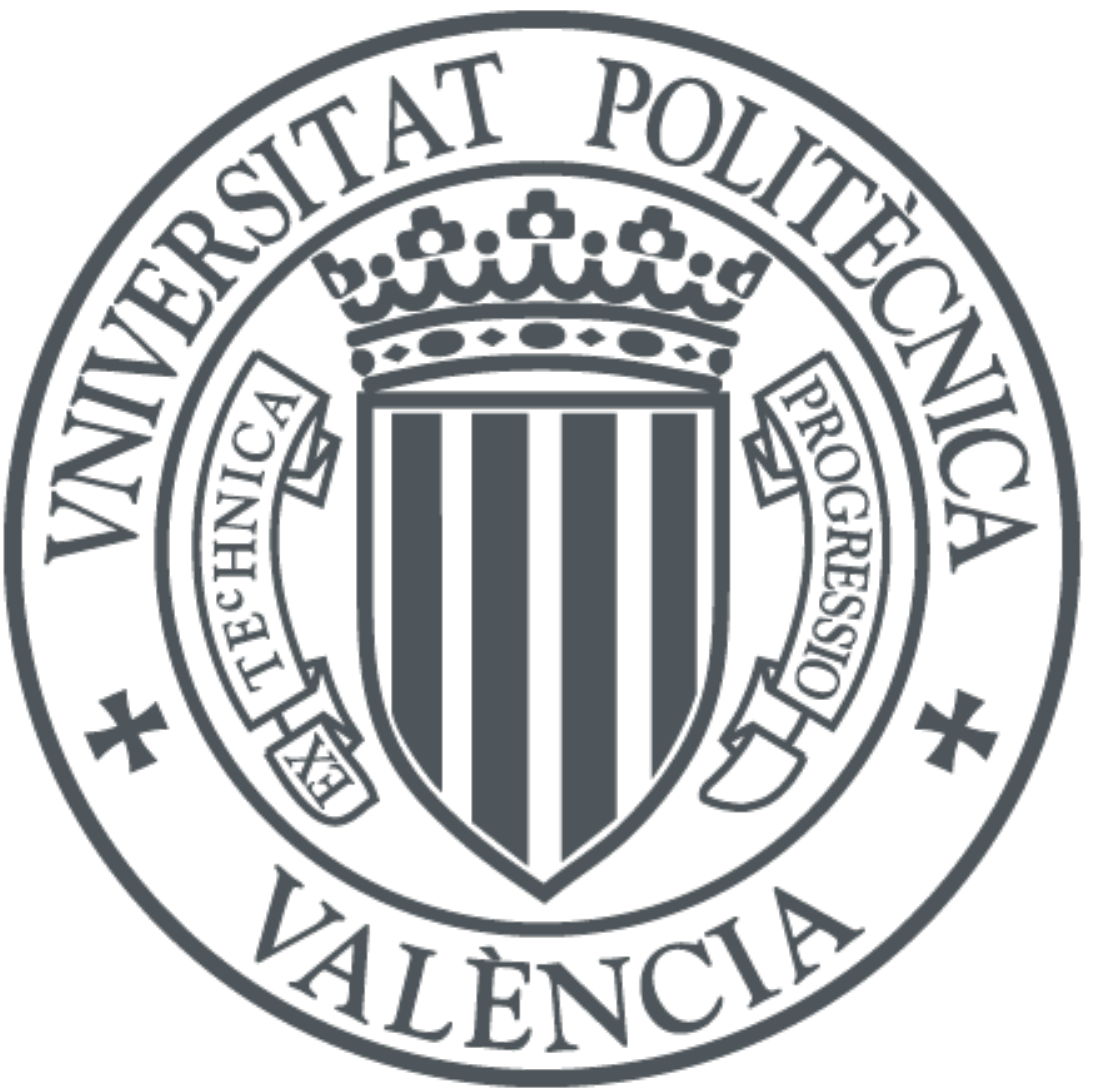

The final publication is available at

http://doi.org/10.1007/s00521-010-0396-2

Copyright SPRINGER

Additional Information 


\title{
Computer-Based Detection and Classification of Flaws in Citrus Fruits
}

\author{
Jose J. Lopez • Maximo Cobos • Emanuel \\ Aguilera
}

Received: date / Accepted: date

\begin{abstract}
In this paper, a system for quality control in citrus fruits is presented. In current citrus manufacturing industries, calliper and color are successfully used for the automatic classification of fruits using vision systems. However, the detection of flaws in the citrus surface is carried out by means of human inspection. In this work, a computer vision system capable of detecting defects in the citrus peel and also classifying the type of flaw is presented. First, a review of citrus illnesses has been carried out in order to build a database of digitalized oranges classified by the kind of fault, which is used as a training set. The segmentation of faulty zones is performed by applying the Sobel gradient to the image. Afterwards, color and texture features of the flaw are extracted considering different color spaces, some of them related to high order statistics. Several techniques have been employed for classification purposes: Euler distance to a prototype, to the nearest neighbor and k-nearest neighbors. Additionally, a three layer neural network has been tested and compared, obtaining promising results.
\end{abstract}

Keywords Computer vision · Automatic inspection system · Texture analysis segmentation · Quality control

\section{Introduction}

The citrus industry in Spain is very important, being one of the biggest ones in the world regarding production, with about 6 million tons in the year 2008. This quantity includes all the range of citrus: orange, lemon, grapefruit and a great number of tangerine varieties. Today, machine vision has been introduced in many industrial applications for citrus processing, allowing the automation of tasks performed so far by human operators. These systems can work faster than manual procedures, but not better in all the cases. Although most of the sorting and packing stages in packing houses

Jose J. Lopez

Universidad Politécnica de Valencia

Institute of Telecommunications and Multimedia Applications

Camino de Vera s/n. 46022, Valencia, Spain

Tel.: +34-963-879714

Fax: +34-963-879583

E-mail: jjlopez@dcom.upv.es 
are automated, workers are still needed to discard the fruits that present defects in their peel. The quality of the peel in fruits, which should be free from defects, is one of the most influential factors in the price of fresh fruit. The reason lies in the association that consumers make between good appearance and quality.

Considerable effort has been made in the field of machine-vision-based defect sorting and grading for different fruits as citrus [1][2] , apples [14][11], olives [5], potatoes [10], other fruits and industries [4]. Nowadays, the increase in computer power at affordable prices and the introduction of multiple core processors allows to process a considerable amount of images in a short time and to use more complex algorithms. A very important part of the process is calyx localization, mainly for two reasons. On one hand, it is needed to distinguish the calyx (that it is not a defect) from other peel defects [12]. On the other hand, there is an increasing demand on automatic systems for rotating the fruit to the correct calyx-relative position. This makes possible to use other fruit processing systems, like industrial peelers.

As commented above, there exist other references related to the detection of defects in the citrus peel [1]. However, in these works, the classifier is only capable to inform whether there is a defect on the peel or not, thus disregarding the information about the defect type. As an extra advantage, it is very interesting to distinguish between different defect types, since this can help to decide on the most suitable actions that should be carried out with the faulty piece of fruit. In particular, some defects do not forbid the fruit to be taken to the market, but they impede it to belong to a higher category. On the other hand, other defects make the fruit be directed to juice manufacturing or, in case of a serious defect, the fruit is directly thrown away.

\subsection{Objectives}

The aim of this work is to develop several image analysis algorithms in order to classify different surface defects in citrus, including the calyx detection. This objective is very ambitious, since the defect type in fruits peel can be related to a high number of different diseases, such as fungus, insects bites or other animals attacks. Moreover, defect types are also dependent on the geographical region where the citrus is grown. In order to establish some limitations, we have focused on a finite and affordable number of existing types. To achieve this objective, a system made up of four steps is proposed:

- First, a segmentation stage based on boundary detection using a Sobel gradient mask for defect localization is explained.

- The second stage implies the transformation of the image into several color spaces. In this paper, the RGB and HSV color models have been considered.

- The third stage is the feature extraction of the defect area found. Five properties on each color component are proposed to distinguish between different peel faults.

- In the last stage, two classification techniques are applied over the features: distancebased classifiers and neural networks.

Previously to these three stages, it will be necessary to create an image data base containing all the defect types manually classified by an expert. 


\section{System Set-Up Overview and Citrus Defect Database}

Although the final aim of the system here presented is to be included in a conveyor belt, for all the experiments conducted in this work, the fruits were kept static inside an inspector chamber. This had diffuse lighting controlled by our lab. In order to obtain images from all the orange surface, it was manually rotated for obtaining 4 different captures, covering the $100 \%$ of the surface. However, when moving from laboratory conditions to a real industry situation, it will be necessary to consider that the fruits are carried by a conveyor-belt from the unload area to the classification area. The camera will be placed at some point of the conveyor.

A common shutter speed of 1000 can be employed without blur degradation. However, since high shutter speeds are employed, high illumination is needed. To avoid the problems caused in the segmentation step by highlights on the fruit surface, we used diffused light. White bulbs where used better than transparent ones because in the first the filament is hidden. Approximately $600 \mathrm{~W}$ were needed to provide the correct illumination. Fluorescent light was discarded because of the noise it produced. This was checked in a real industry conveyor belt observing that, since this artificial light is not really white, the camera had to be previously equalized with white balance control. The cameras employed where digital cameras with direct digital output via Firewire. The resolution employed was $640 \times 480$ pixels at 256 levels per channel $(\mathrm{R}, \mathrm{G}, \mathrm{B})$.

\subsection{Citrus Defect Database}

A database of the most common different defects in the eastern region of Spain was built. A set of 150 oranges from "navel-late" and "valencia-late" varieties were digitalized in the laboratory. Each orange was captured in 10 different positions covering the full surface, obtaining a set of 1500 images.

Each orange was classified by an expert and afterwards labelled according to the defect type its peel had. This data base constituted the training set for the classifying stage. The defects that were identified and that were considered for present work are the following:

- A. "Wind scar" : the wind can produce lights lesions due to friction with branches.

- B. "Stem-end breakdown" or "ageing".

- C. "Snail bites".

- D. "Thrips scar".

- E. "Scale injury". Where we can find "Parlatoria ziziphi", "Parlatori pergandei", "Chrysomphalus dictiospermi", etc.

- F. "Mediterranean fruit fly".

- G. Others faults that can be located from visual inspection.

- H. Stem-calyx. The location of the calyx is an important step, because it must not be confused with one defect. Also, the zone where a calyx was removed must not be confused either. These considerations can be found in some other works [9][12].

Figure 1 shows different examples of the analyzed defect groups (including the Stem-calyx). 
A. Wind Scar

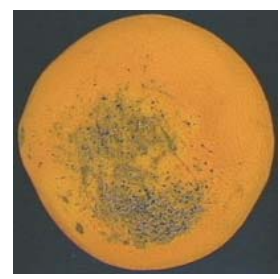

E. Scale injury

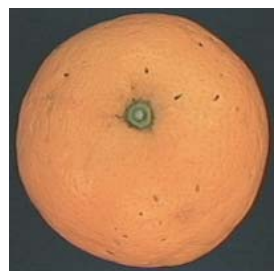

B. Ageing

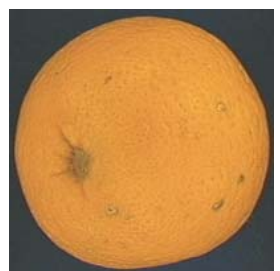

F. Medfly

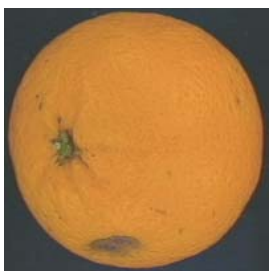

C. Snail Bites

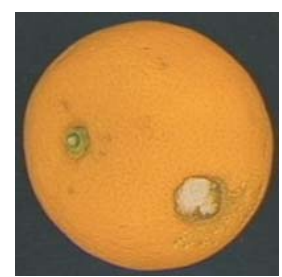

G. Other

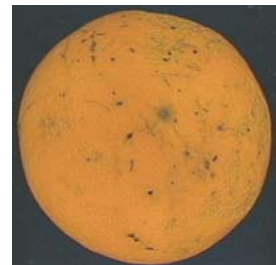

D. Thrips scar

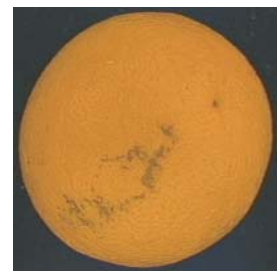

H. Calyx

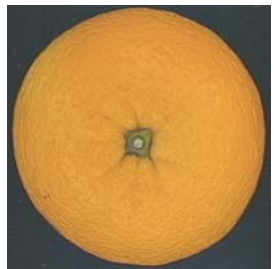

Fig. 1 Example of different types of peel defects.

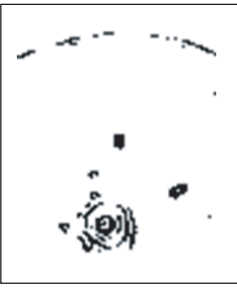

(a)

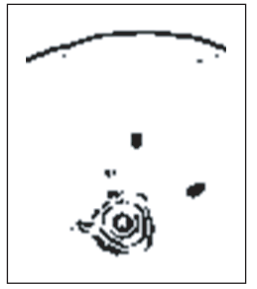

(b)

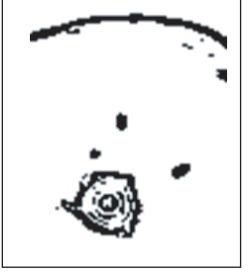

(c)

Fig. 2 Images after Sobel gradient mask and thresholding. a)Blue, b)Green and c) Red.

\section{Proposed Processing}

\subsection{Defect Area Segmentation}

Assuming a digitalized RGB color image of a citric, the first objective is the segmentation of the peel defect area. We propose a boundary detection method for this task based on a Sobel gradient mask:

$$
\begin{gathered}
G_{y}=\left[\begin{array}{ccc}
-1 & -2 & -1 \\
0 & 0 & 0 \\
1 & 2 & 1
\end{array}\right], \quad G_{x}=\left[\begin{array}{lll}
-1 & 0 & 1 \\
-2 & 0 & 2 \\
-1 & 0 & 1
\end{array}\right] \\
\text { Gradient }=\sqrt{\left(\left|G_{x}\right|+\left|G_{y}\right|\right)} .
\end{gathered}
$$

For the most part of fruits, as oranges, the components $\mathrm{R}$ and $\mathrm{G}$ of the surface color are always stronger than the B component, as it can be seen in Figure 2. However, the $\mathrm{G}$ component can sometimes provide wrong cues about the defect of the fruit, because the mature state of different parts of the fruit can be interpreted as boundary. So it seems reasonable to use the $\mathrm{R}$ component in this phase. 


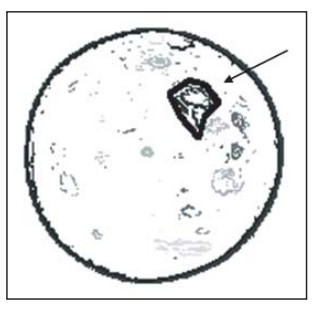

(a)

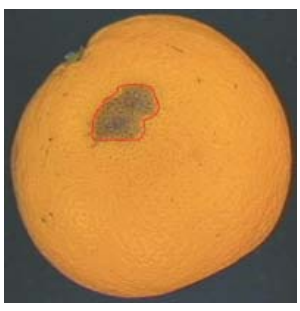

(b)

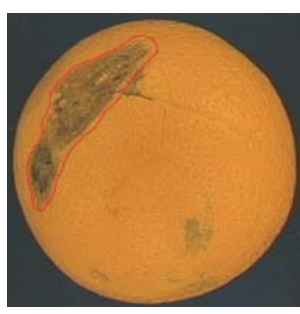

(c)

Fig. 3 (a) Polygonal approximation of one faulty zone. Each grey level stands for a different border. (b-c) Segmentation area for two types of defects.

After boundary detection, the resulting image must be post-processed in order to recognize the different boundaries (orange contour and faults outline). Each edge is assigned to a label. To assign a new edge, neighborhood and gradient threshold criterions are used. The process of segmenting the orange from the background is not difficult. We only consider the main edge, thus the background can be non uniform as the conveyor belt. Since each defect zone is limited by a labelled edge, we can locate it inside a polygon approximation, see Figure 3(a), so that we avoid problems of edge discontinuity. To perform this task, a slightly modified version of the well-known split method by Douglas and Peucker [6] was used. Although other sophisticated methods can be applied [8], it was experimentally tested that the change in the computed feature vectors was very small $(<2 \%)$. As it will be seen along the next subsections, these features are based on color and texture content, and therefore, slight variations in shape are not critical.

Also we can determine an approximation of the percent of damaged zone over the total orange surface. Finally, Figure 3(b-c) shows the result of the segmentation area marked on the original image by a red line. It can be seen that the segmentation is very precise. This fact is very important for the application of the later classification stage.

\subsection{Color space transformation}

Once the defect area has been isolated, the main features are extracted from the color information of its pixels. Therefore, the first step is to transform the color space into a more useful one that makes easier the defect discrimination task.

Commonly used CCD cameras work under a RGB space. This space refers to the intensity of the three primary colors (red, green and blue) in which a certain color can be decomposed (Figure 4(a)). Therefore, although this color model is based on simple additive synthesis, it is not completely intuitive from a perceptually point of view.

A more interesting color space, which is perceptually uniform, is the HSV (Hue, Saturation, Value) model. In this model, the components are expressed in cylindrical coordinates, where the proximity of the values implies color similarity (Figure 4(b)). Hue is represented as an angle between $0^{\circ}$ and $360^{\circ}$, each angle corresponding to one color. Saturation represents the distance to the black-white brightness axis (cone axis). Value indicates the brightness of the color and represents the height along the black-white axis. 


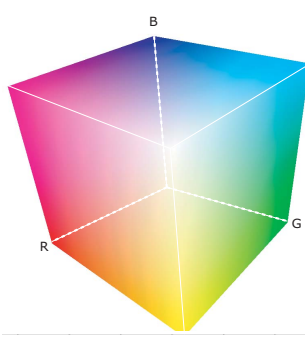

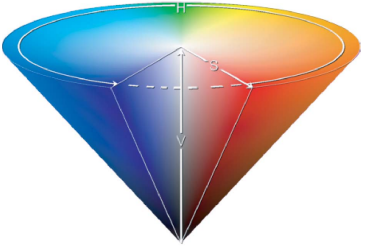

Fig. 4 Color spaces. (a) RGB color model. (b) HSV color model.

The HSV model can be obtained by means of a non-linear transformation of the RGB space:

$H(R, G, B)=\left\{\begin{array}{lll}\text { non-defined } & \text { if } & \max (R, G, B)=\min (R, G, B) \\ 60^{\circ} \cdot \frac{G-B}{\max (R, G, B)-\min (R, G, B)} & \text { if } & \max (R, G, B)=R, G \geq B \\ 60^{\circ} \cdot \frac{G-B}{\max (R, G, B)-\min (R, G, B)}+360^{\circ} & \text { if } & \max (R, G, B)=R, G<B \\ 60^{\circ} \cdot \frac{B-R}{\max (R, G, B)-\min (R, G, B)}+120^{\circ} & \text { if } & \max (R, G, B)=G \\ 60^{\circ} \cdot \frac{R-G}{\max (R, G, B)-\min (R, G, B)}+240^{\circ} & \text { if } & \max (R, G, B)=B\end{array}\right.$
$S(R, G, B)=\left\{\begin{array}{lll}0 & \max (R, G, B)=0 \\ 1-\frac{\min (R, G, B)}{\max (R, G, B)} & \text { otherwise }\end{array}\right.$
$V(R, G, B)=\max (R, G, B)$

In the following, both color spaces (RGB and HSV) will be used for the feature extraction task described in the next section. As an example, the components of an orange image in both spaces are shown in Figure 5. Note that, if the lighting conditions involved in the system are not controlled, special care must be taken for not taking into account brightness changes and avoid illumination dependance. This can be achieved by applying a normalization step [7].

\subsection{Feature Extraction}

In this second stage, we propose five features that let us distinguish between different types of peel defect regions:

- Mean:

$$
\mu\left[C_{j}\right]=\sum_{l=0}^{255} l \cdot P\left(l, C_{j}\right) \quad j=1,2,3,
$$

where $C_{j}$ indicates the $j$-th component of the color space and $P\left(l, C_{j}\right)$ the histogram of the color component [13].

- Range

$$
R\left[C_{j}\right]=\frac{\max _{0<l<255}\left\{P\left(l, C_{j}\right) \neq 0\right\}-\min _{0<l<255}\left\{P\left(l, C_{j}\right) \neq 0\right\}}{255} \quad j=1,2,3 .
$$



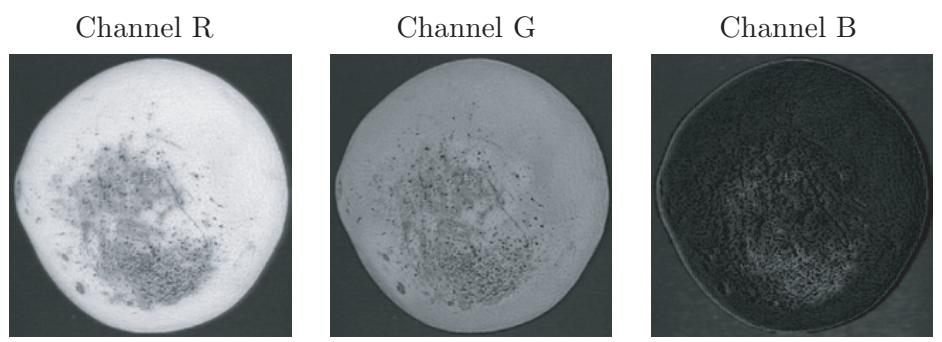

(a)

Channel $\mathrm{H}$
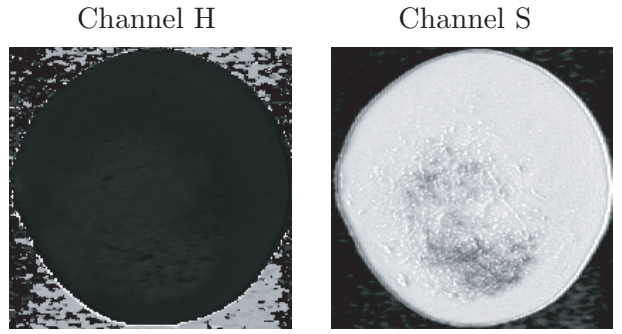

Channel V

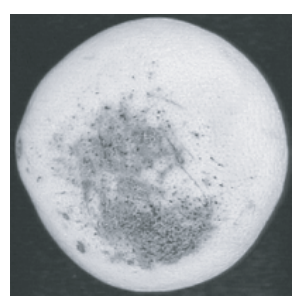

(b)

Fig. 5 Color components of an orange image. (a) RGB components. (b) HSV components.

- Variance

$$
\sigma^{2}\left[C_{j}\right]=\sum_{l=0}^{255}\left(l-\mu\left[C_{j}\right]\right)^{2} \cdot P\left(l, C_{j}\right) \quad j=1,2,3 .
$$

- Skewness

$$
s\left[C_{j}\right]=\sum_{l=0}^{255}\left(l-\mu\left[C_{j}\right]\right)^{3} \cdot P\left(l, C_{j}\right) / \sigma^{3 / 2} \quad j=1,2,3 .
$$

- Kurtosis

$$
k\left[C_{j}\right]=\sum_{l=0}^{255}\left(l-\mu\left[C_{j}\right]\right)^{4} \cdot P\left(l, C_{j}\right) / \sigma^{2} \quad j=1,2,3 .
$$

Mean and range are simple descriptors based on first order statistics, while the others are based on higher order statistics. These features are computed for each color component $(j=1,2,3)$ so we will have a vector of 15 features for the next classification stage.

\subsection{Classification}

After using the previously presented segmentation techniques, some different faulty areas are obtained. For these areas, the above considered features are extracted in order to conform the feature vector. A major task after the feature extraction is to classify the segmented area into one of several fault categories, including the calyx detection. For this purpose, we are employing mainly two classification techniques: distance-based classifiers and neural networks. 


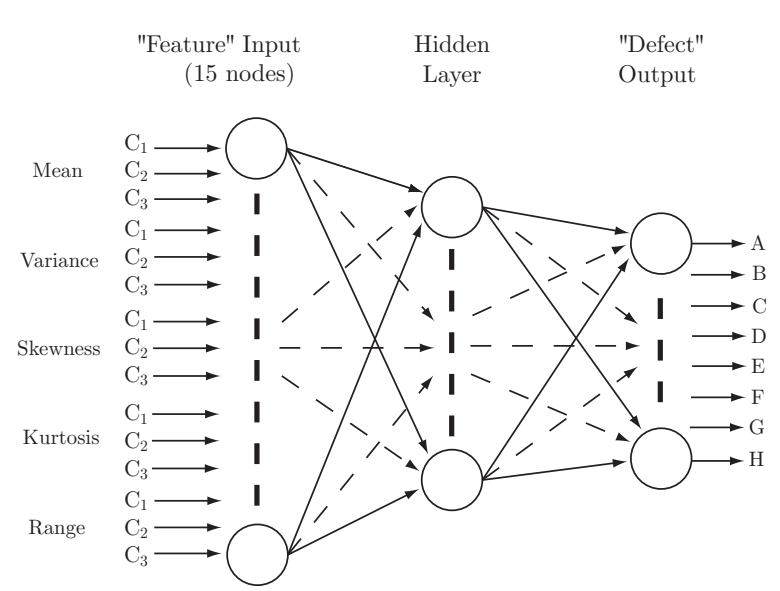

Fig. 6 Three layer neural network employed in the classifier.

With distance-based classifiers we are employing three different methods: the mininum distance (MD), the nearest neighbour (NN) and the k-nearest neighbours (k-NN). While the first one selects the unique prototype minimum distance, the second one selects the nearest training sample. The third one selects the k-nearest training samples. The value of $\mathrm{k}$ was experimentally selected using 10 -fold cross-validation, obtaining an optimum value of $k=6$. Since we are working with more than 2 classes, it is not critical to select an odd number of neighbors. Also, different feature combinations are used. As commented in the last subsection, the first test uses mean and range, the second test uses variance, skewness and kurtosis, and a mixture of all these features is used in the third test.

A feed-forward backpropagation neural network (Net) is the other technique being used to classify the different fault categories. The network model designed has 15 input nodes corresponding to the extracted features of the image components $\left(C_{1}, C_{2}, C_{3}\right)$ for the selected color space. Then, it has one hidden layer with ten nodes. And finally it has eight output nodes, one for each kind of citrus fault, included the calyx detection, see Figure 6. All the functions to activate each neurone are considered to be sigmoids. The neural network was trained by backpropagation by gradient descent until reaching a correct classification [3].

\section{Test and Results}

Using the system discussed before, we digitalized another different set of 180 oranges from the "navel-late" and "valencia-late" varieties. In this case each orange was digitalized only in 4 different positions simulating a conveyor belt, obtaining a set of 720 images. Also in this case, the expert previously classified the type of defect in order to evaluate the performance achieved by the developed automatic system. Next, the proposed computer vision software was executed and feeded with the second database in order to classify this new set of oranges. Several classification tasks were performed using the four proposed methods and the training data set. It was also studied how the performance was affected depending on the chosen features. With this goal, three different feature sets were created, using one or more feature vectors (FV) with each of the proposed classifiers. 
- FV1: (Mean, Range)

- FV2: (Variance, Skewness, Kurtosis)

- FV3: (Mean, Range, Variance, Skewness, Kurtosis)

Note that the above feature vectors were selected according to the statistics order. FV1 is composed only of first order statistics, providing basic information about the color content of the segmented area. On the other hand, FV2 contains statistics of higher order, providing more detailed information related to the texture of the defect area. Finally, FV3 contains all the proposed features, combining FV1 and FV2 into a single vector.

The results are shown in Table 1 and Table 2. Columns reflect the classification method: and the feature vector used. Rows reflect the kind of citrus defect as described in section 2.1. Each cell shows the correct detection rate. Analyzing both table values, it can be observed that, in general, the use of more features results in an increased performance, which justifies the introduction of high order features present in FV2. However, results are slightly better for features based on the HSV color space. This probably a consequence of the non-linear transformation of the RGB space, which may represent better the small color variability between samples. This was also noted by other authors [2][7]. Regarding the classification algorithm, it is also noticeable that the more sophisticated ones (k-Nearest Neighbors and Neural Network) produced considerably better results.

\begin{tabular}{cccccccccc}
\hline & \multicolumn{3}{c}{ Prot. } & \multicolumn{2}{c}{ NN } & \multicolumn{3}{c}{ k-NN } \\
\hline & FV1 & FV2 & FV3 & FV1 & FV2 & FV1 & FV2 & FV3 \\
\hline A & $<50$ & $<50$ & $<50$ & $<50$ & 62.5 & $<50$ & 67.3 & 83.7 & FV3 \\
B & $<50$ & 71.4 & 60.2 & 100 & 100 & 50 & 100 & 100 & 94.2 \\
C & 71.4 & $<50$ & 57.1 & 78.5 & 57.1 & 78.5 & 50 & 92.8 & 100 \\
D & 77 & $<50$ & 69.2 & 61.5 & 69.2 & 62.6 & 84.6 & 92.3 & 94.2 \\
E & 94.1 & 66.6 & 100 & $<50$ & 66.6 & $<50$ & $<50$ & $<50$ & 72.3 \\
F & $<50$ & $<50$ & $<50$ & 53.8 & 69.2 & 77.1 & 52.3 & 84.6 & 63.2 \\
G & 66.6 & $<50$ & $<50$ & $<50$ & 50 & $<50$ & $<50$ & 75 & 72.5 \\
H & $<50$ & $<50$ & $<50$ & $<50$ & 58.3 & $<50$ & 58.3 & 66.8 & 69.3 \\
\hline
\end{tabular}

Table 1 Overall percentage correct classification using RGB color components.

\section{Conclusions and Future Work}

A system for quality inspection in the citrus industry has been presented. In its design, a special effort has been made for building a reliable training data set including many types of peel flaws. This defect identification task has been carried out by experts in the field, providing our system with very useful information successfully employed in the classification task. The system is able to run in real time for a typical conveyor belt speed of $0.1 \mathrm{~s}$. In our implementation, a quad-core state-of-the-art personal computer was used. Moreover, a study of the influence of the color space used has also been carried out. Future work will be focused on improving the performance by introducing new statistical-based features not only related to color. For example, the shape and size 


\begin{tabular}{ccccccccccc}
\hline & \multicolumn{3}{c}{ Prot. } & \multicolumn{2}{c}{ NN } & \multicolumn{3}{c}{ k-NN } \\
\hline & FV1 & FV2 & FV3 & FV1 & FV2 & FV1 & FV2 & FV3 & FV3 \\
\hline A & $<50$ & $<50$ & $<50$ & 53.3 & 65.7 & 54.3 & 68.9 & 88.8 & 95.1 \\
B & 51.1 & 70.7 & 63.3 & 100 & 100 & 60.3 & 100 & 100 & 100 \\
C & 75.3 & 55.4 & 59.5 & 77.7 & 58.8 & 79.3 & 55.1 & 94.1 & 95.5 \\
D & 77.3 & $<50$ & 69.1 & 60.9 & 70.1 & 64.1 & 84.9 & 92.8 & 75.4 \\
E & 93.8 & 68.7 & 100 & $<50$ & 67.3 & $<50$ & 55.3 & $<50$ & 62.8 \\
F & $<50$ & $<50$ & $<50$ & 57.6 & 72.4 & 78.8 & 55.1 & 85.1 & 77.1 \\
G & 68.5 & $<50$ & 51.3 & 51.7 & 52.3 & 53.3 & 61.1 & 74.3 & 69.5 \\
H & $<50$ & $<50$ & 54.3 & $<50$ & 59.9 & 53.7 & 60.0 & 67.0 & 64.0 \\
\hline
\end{tabular}

Table 2 Overall percentage correct classification using HSV color components.

of defects is being currently exploited as a possible improvement line. Moreover, most of the classifiers used in this work were based on distance. Besides neural networks, which have been already used, it will be interesting to use more sophisticated classifiers, such as support vector machines (SVM), random forests and boosting classifiers.

\section{References}

1. Blasco, J., Aleixos, J., Molto, E.: Computer vision detection of peel defects in citrus by means of a region oriented segmentation. Journal of Food Engineering 81, 535-543 (2007)

2. Blasco, J., Aleixos, N., Gomez, J., Molto, E.: Citrus sorting by identification of the most common defects using multispectral computer vision. Journal of Food Engineering $\mathbf{8 3}$, 384-391 (2007)

3. Bryson, A.E., Ho, Y.C.: Applied optimal control: optimization, estimation, and control. Xerox College Publishing (1969)

4. Conners, R.W.e.a.: Identifying and locating surface defects in wood. IEEE Transactions on Pattern Analysis and Machine Intelligence 5, 573-583 (1983)

5. Diaz, R., Gil, L., Serrano, C., Blasco, M., Molto, E., Blasco, J.: Comparison of three algorithms in the classification of table olives by means of computer vision. Journal of Food Engineering 61, 101-107 (2004)

6. Douglas, D.H., Peucker, T.K.: Algorithm for the reduction of the number of points required to represent a line or its caricature. The Canadian Cartographer 10(2), 112-122 (1973)

7. Du, C.J., Sun, D.W.: Comparison of three methods for classification of pizza topping using different colour space transformations. Journal of Food Engineering 68, 277-287 (2005)

8. Kolesnikov, A.: Efficient algorithms for vectorization and polygonal approximation. Ph.D. thesis, University of Joensuu, Finland (2003)

9. Molto, E.e.a.: A computer vision system for inspecting citrus,peaches and apples. In: Proceedings of VII National Symposium on Pattern Recognition and Image Analysis, pp. 121-126. Sabadell, Spain (1997)

10. Muir, A.Y., Porteus, R.L., Wastie, R.L.: Experiments in the detection of incipient diseases in potato tubers by optical methods. Journal of Agricultural Engineering Research 27, 131-138 (1982)

11. Qingzhong Li, e.a.: Computer vision based system for apple surface defect detection. computer and electronics in agriculture. Computer and Electronics in Agriculture 36, 215-223 (2002)

12. Ruiz, L.A., Molto, E., Juste, F., Pla, F., Valiente, R.: Location and characterization of the stemcalyx area on oranges by computer vision. Journal of Agricultural Engineering Research 64, 165-172 (1996)

13. Tan, T.S.C., Kittler, J.: Colour texture analysis using colour histogram. IEEE Proc.-Vis. Image Signal Process 141, 403-412 (1994)

14. Wen, Z., Tao, Y.: Building a rule-based machine-vision system for defect inspection on apple sorting and packing lines. Expert Systems with Applications 16, 307-313 (1999) 